\title{
Psychiatric comorbidity as a risk factor for the mortality of people with bulimia nervosa
}

\author{
Hubertus Himmerich $^{1,2} \cdot$ Matthew Hotopf $^{1,2} \cdot$ Hitesh Shetty $^{2} \cdot$ Ulrike Schmidt $^{1,2} \cdot$ Janet Treasure $^{1,2}$. \\ Richard D. Hayes ${ }^{1} \cdot$ Robert Stewart $^{1,2} \cdot$ Chin-Kuo Chang ${ }^{1,2,3}$
}

Received: 28 May 2018 / Accepted: 4 February 2019 / Published online: 11 February 2019

(c) The Author(s) 2019

\begin{abstract}
Background Bulimia nervosa (BN) is associated with increased mortality. Frequent comorbidities of BN include substance use disorders, affective disorders and personality disorders (PD). These comorbidities may add an additional risk for mortality. Methods We investigated the influence of these psychiatric comorbidities on all-cause mortality with demographic and socioeconomic factors considered as confounders over an observation period from January 2007 to March 2016 for 1501 people with BN using anonymised health records data from the South London and Maudsley NHS Foundation Trust (SLaM), retrieved through its Clinical Records Interactive Search (CRIS) data resource. Mortality was ascertained through monthly linkages to the nationwide tracing system administered by the Office for National Statistics (ONS). We used Cox proportional hazards regression to calculate hazard ratios (HRs) with 95\% confidence intervals (CIs). Multivariable analyses were also performed to estimate effects when controlling for confounding of age, sex, ethnicity, borough, marital status and deprivation score.

Results A total of 18 patients with BN died during the observation period. The standardised mortality ratio (SMR) for our study cohort (against the population of England and Wales in 2012 as a standard) was 2.52 (95\% CI 1.49-3.97). Cox regressions revealed significant associations of mortality with older age and male gender. Comorbid PD (HR: 3.36; 95\% CI 1.05-10.73) was significantly associated with all-cause mortality, even after controlling for demographic and socioeconomic covariates.

Conclusions These results highlight increased mortality in patients with $\mathrm{BN}$ and the importance of recognising and treating PDs in patients with BN.
\end{abstract}

Keywords Bulimia nervosa $\cdot$ Eating disorder $\cdot$ Psychiatric comorbidities $\cdot$ Affective disorders $\cdot$ Substance use disorders . Personality disorder

\section{Introduction}

\section{Bulimia nervosa}

Electronic supplementary material The online version of this article (https://doi.org/10.1007/s00127-019-01667-0) contains supplementary material, which is available to authorized users.

Chin-Kuo Chang

ckchang@utaipei.edu.tw

1 Department of Psychological Medicine, King's College London, London, UK

2 South London and Maudsley NHS Foundation Trust, London, UK

3 Department of Health and Welfare, University of Taipei, No. 101, Sec. 2, Jhongcheng Rd, Shilin District, Taipei 111, Taiwan
Bulimia nervosa $(\mathrm{BN})$ is an eating disorder $(\mathrm{ED})$ that was included in the 3rd edition of the Diagnostic and Statistical Manual of Mental Disorders (DSM-III) in 1980 [1] after its description by Gerald Russell the year before [2]. The current main criteria for the diagnosis of $\mathrm{BN}$ according to DSM-5 are recurrent binges, i.e. consumption of an unusually large amount of food within a short time interval, compensatory behaviours such as vomiting, excessive physical activity or fasting at least once a week for 3 months, and excessive preoccupation with shape and weight [3]. Even though the current version of DSM, the DSM-5 is the most 
contemporary classification system (issued in 2013 [3]), its use is restricted to research and psychiatric practice in the United States of America (USA). Most countries such as the United Kingdom (UK) use chapter V of the10th revision of the International Statistical Classification of Diseases and Related Health Problems (ICD-10; issued in 1992 [4]) to diagnose psychiatric disorders in clinical practice. Diagnostic criteria for BN according to ICD-10, however, are similar to DSM-5 criteria and include recurrent episodes of overeating in which large amounts of food are consumed in short periods of time, a persistent preoccupation with eating and a strong desire to eat, attempts to counteract the fattening effects of food and a self-perception of being too fat [4].

$\mathrm{BN}$ occurs in $1-2 \%$ of women with a sex ratio of men to women of about $1: 10[5,6]$. It is often associated with affective disorders (AD) [7], substance use disorders (SUD) [8-11] and personality disorders (PD) [12-14]. It has been hypothesized that these comorbidities-specifically SUD and PD-develop on the basis of an increased impulsivity and affective instability in patients with BN [15-18]. This view is supported by findings that borderline PD traits are particularly common among patients with $\mathrm{BN}$ [19].

\section{Mortality in BN}

Mortality rates are reported to be elevated in patients with BN. For instance, in a recently published German study on long-term mortality in EDs which included 1930 people BN [20], the standardised mortality ratio (SMR) for people with $\mathrm{BN}$ was estimated at 1.49 indicating that the observed number of deaths in the $\mathrm{BN}$ population was about 1.5 times higher than expected in the general population standardised for sex and age. For this study, the research team had included people during their inpatient treatment in a hospital for behavioural medicine with a subspecialty for EDs.

Another example of a study looking at mortality rates has been published by Hoang et al. [21]. They used a dataset of the English national Hospital Episode Statistics (HES), linked with data from death records provided by the Office for National Statistics (ONS). They included all cases, where an ED was recorded as the main diagnosis on discharge of a treatment in hospital. They identified 838 people with $\mathrm{BN}$ and obtained an SMR of 4.1 for these patients [21]. This was probably a patient cohort of more severely affected people, as BN patients are usually not treated as inpatients in the UK, because the guidelines of UK's National Institute for Health and Care Excellence (NICE) for the recognition and treatment of EDs specify that patients with EDs should only be admitted, if their physical health is severely compromised in such a way that they need medical stabilisation or refeeding [22]. In Germany, by contrast, there is a tradition of treating patients with EDs in psychosomatic hospitals, and the so-called $\mathrm{S} 3$ guidelines for the diagnosis and treatment of EDs specify comparably low-threshold indicators for inpatient therapy for patients with $\mathrm{BN}$ such as a lack of local outpatient treatment opportunities, the severity of the disorder, severe conflicts in the family and the social environment [23].

In a former systematic review and meta-analysis that included 12 quantitative studies and a sum of 2,585 individuals with BN across different countries, SMR was estimated at 1.9 [24] which might be a quite balanced SMR estimation.

There are different potential reasons, why BN is associated with increased mortality. Broadly speaking, increased mortality in this patient group might be related to the physical complications of BN [25] or due to a higher risk of suicidality [26, 27]. Potentially life-threatening complications of $B N$ include gastrointestinal, endocrine and renal disturbances which can lead to severe electrolyte derangements and cardiac arrhythmias [25]. Among others, important factors contributing to the increased risk of suicide in people with BN could be the high level of impulsiveness [26], hopelessness [28] and — as already mentioned - comorbidities such as SUDs, PDs and ADs, like unipolar depression [28], which themselves are also associated with increased mortality [7-14, 29-33]. In the literature, SUDs, PDs, ADs, and anxiety disorders were found the most frequent mental disorders comorbid to $\mathrm{BN}$ [34-36]. Among those four, SUDs have specifically been reported to contribute to mortality in BN [37, 38], and PDs [39] as well as ADs [40] have each been shown to be associated with increased mortality - independently from the presence of $\mathrm{BN}$, whereas a recent meta-analysis did not reveal any evidence of an increased mortality risk among anxious study participants. The increased mortality in patients with anxiety disorders previously found in small study samples has been considered to be most probably due to comorbid depression [41]. Therefore, we decided to focus on SUDs, PDs and ADs as the most promising potential factors associated with increased morbidity in patients with BN. Other demographic variables which have been associated with mortality in patients with EDs or ADs include age, gender, marital status, socioeconomic deprivation and ethnicity $[42,43]$.

\section{Aim of the study}

In this study, we sought to investigate the influence of major psychiatric comorbidities on all-cause mortality in people with $\mathrm{BN}$ in a case register for a large secondary mental healthcare service provider in southeast London, UK, with confounding from demographic and socioeconomic factors considered. Our hypothesis for this investigation was that AD, SUD and PD would have independent effects on increased mortality in people with BN. 


\section{Methods}

\section{Setting and study design}

A retrospective cohort study was conducted in people with a diagnosis of BN. The BN cohort for the analysis was obtained from the South London and Maudsley National Health Service (NHS) Foundation Trust (SLaM) which provides near-monopoly secondary mental healthcare services to approximate 1.36 million residents in southeast London. Patients involved in this study were mainly from the London boroughs of Lambeth, Croydon, Lewisham, Southwark, Bromley, Bexley, and Greenwich. SLaM has used fully electronic records in all its services since 2016 and its NIHRfunded Biomedical Research Centre (BRC) supports an infrastructure for rendering its anonymised records available for research, using the its Clinical Records Interactive Search (CRIS) platform, established in 2008 [44, 45].

\section{Inclusion criteria}

The analysed cohort was extracted via CRIS, consisting of individuals who received an International Classification of Diseases (ICD-10) diagnosis [4] of BN (F50.2) within or before the observation window from 1 January 2007 to 31 March 2016. Structured information on diagnosis from drop-down fields in the source record was supplemented by information extracted from open-text fields using a bespoke algorithm generated using the Generalised Architecture for Text Engineering (GATE) software [44]. The exposure of interest was a further documented diagnosis of SUD (F10-F19), AD (F31-33) or PD (F60) at any stage during or before the observation window extracted from CRIS using identical procedures.

\section{Primary outcome}

The outcome of interest in this analysis was all-cause mortality occurring from 1 January 2007 to 31 March 2016. Information for each death was collected through nationwide tracing using data from the Office for National Statistics (ONS) linked to the SLaM database on a monthly basis. In the UK, all death certifications are linked by NHS number (a unique identifier for each UK NHS service user) to all healthcare providers.

\section{Covariates}

Date of birth (year and month), gender, ethnicity, marital status and residing borough were retrieved from CRIS database. Age in years was calculated from the individual's first
$\mathrm{BN}$ diagnosis date in the observation window or on 1 January 2007 if the BN diagnosis preceded the observation window. Recorded ethnicity was classified into three categories: 'white' (including 'British', 'any other white background' and 'Irish'), 'black' (including 'African', 'any other black background' and 'Caribbean'), and 'others/unknown/mixed'. Recorded marital status was classified into four groups: 'married' (including 'cohabitation', 'married' and 'married/ civil partnership'), 'single', 'divorced' (including 'divorced', 'civil partnership dissolved' and 'separated'), and 'others' (including 'others' and 'not known'). Borough was classified by the subject's residence: Lambeth, Croydon, Lewisham, Southwark, Bromley, Bexley, and Greenwich, defined by their primary care trust. Multiple deprivation score is a small-area-level measure of socioeconomic status, based on the individual's address closest to the diagnosis of $\mathrm{BN}$ in the observation window (or 1 January 2007 for those with an earlier diagnosis of $\mathrm{BN}$ ), covering the following seven components: 'employment, income, education, health, barriers to housing and services, crime and the living environment' with specific weightings. The Index of Multiple Deprivation core is a well-established measure which has been widely used as a regional indicator for socioeconomic status in previous studies [46-48]. The score is then transformed into percentiles (from 1 to 100) with a higher score, indicating greater deprivation (and thus a lower socioeconomic status in the neighbourhood). In the analysis, deprivation score was grouped into tertiles (33\% percentiles).

\section{Statistical analysis}

Standardised mortality ratios (SMRs) were used to describe case mortality in relation to the general population. Observed number of deaths in the observation window was treated as the numerator and 5-year age and sex bands were defined to calculate expected numbers for the same bands from the population of England and Wales in 2012 according to ONS data. Since the study cohort was followed for up to 9.25 years, multipliers based on the average follow-up period for each corresponding age-and-sex band were applied for estimating general population denominators.

Descriptive statistics on demographic characteristics and psychiatric comorbidities were first carried out for the study cohort. We then used Cox proportional hazards regression to calculate hazard ratios (HRs) with 95\% confidence intervals (CIs) on death as the 'event' for outcome within the observation period. Beginning of the follow-up period was defined as the start of the observation period (1 January 2007) if the first diagnosis of $\mathrm{BN}$ was given before the window; otherwise this was taken as the date of first $\mathrm{BN}$ diagnosis recorded in the window.

Multivariable analyses were also performed to estimate the effect of the considered psychiatric comorbidities as 
major exposure of interest, controlling for confounding of age, sex, ethnicity, borough, marital status and deprivation score. All the statistical analyses were performed using Stata 12.1 (StataCorp, College Station, Texas, USA) and the statistical significance (alpha level) was set at 0.05.

\section{Ethical approval}

Ethical approval was obtained from the Oxfordshire Research Ethics Committee C for the use of CRIS as a source of data for secondary analysis (reference 08/ H0606/71 +5) including the mortality linkage. This study has thus been performed in accordance with the ethical standards laid down in the 1964 Declaration of Helsinki and its later amendments.

\section{Results}

\section{Descriptive statistics}

A total of 1501 individuals diagnosed with BN were identified from the CRIS system, of whom 1431 (94.4\%) were female; the mean age was 28.5 years (standard deviation $=9.5$ years), and the age range was $11.9-64.5$ years old. More detailed information about the age distribution can be found in Table 1. During the observation window, 18 of the 1501 patients died. The mean age of the patients who died in the observation window was 34.9 years old (standard deviation $=12.9$ ). Further detailed descriptive information on age, gender, marital status, the residential borough, ethnicity, social deprivation and comorbidities is given in Table 2.

Table 1 Age distribution of study participants with frequencies of deaths and psychiatric comorbidities by age groups $(\mathrm{N}=1501)$

\begin{tabular}{lllll}
\hline $\begin{array}{l}\text { Age group } \\
\text { (years old) }\end{array}$ & \multicolumn{4}{l}{ Number $\%$ in rows) } \\
\cline { 2 - 5 } & Deaths & SUD & AD & PD \\
\hline $10-14(n=38)$ & $0(0.00 \%)$ & $1(2.63 \%)$ & $5(13.16 \%)$ & $4(10.43 \%)$ \\
$15-19(n=211)$ & $0(0.00 \%)$ & $7(3.32 \%)$ & $42(19.91 \%)$ & $27(12.80 \%)$ \\
$20-24(n=338)$ & $6(1.78 \%)$ & $16(4.73 \%)$ & $40(11.83 \%)$ & $27(7.99 \%)$ \\
$25-29(n=390)$ & $3(0.77 \%)$ & $9(2.31 \%)$ & $56(14.36 \%)$ & $32(8.21 \%)$ \\
$30-34(n=223)$ & $1(0.45 \%)$ & $8(3.59 \%)$ & $32(14.35 \%)$ & $13(5.83 \%)$ \\
$35-39(n=117)$ & $1(0.85 \%)$ & $4(3.42 \%)$ & $24(20.51 \%)$ & $9(7.69 \%)$ \\
$40-44(n=75)$ & $2(2.67 \%)$ & $5(6.67 \%)$ & $17(22.67 \%)$ & $6(8.00 \%)$ \\
$45-49(n=49)$ & $3(6.12 \%)$ & $5(10.20 \%)$ & $8(16.33 \%)$ & $6(12.24 \%)$ \\
$50-54(n=37)$ & $1(2.70 \%)$ & $5(13.51 \%)$ & $9(24.32 \%)$ & $1(2.71 \%)$ \\
$55-59(n=17)$ & $0(0.00 \%)$ & $0(0.00 \%)$ & $7(41.18 \%)$ & $5(29.41 \%)$ \\
$60-64(n=6)$ & $1(16.67 \%)$ & $1(16.67 \%)$ & $2(33.33 \%)$ & $1(16.67 \%)$ \\
\hline
\end{tabular}

$S U D$ substance use disorder, $A D$ affective disorder, $P D$ personality disorder
The recorded causes of death among the 18 people with BN who have died in the observation window were: lethal intoxication from drugs or alcohol $(n=4)$, suicide $(n=3)$, circulatory or heart failure $(n=3)$, respiratory failure $(n=3)$ and cancer $(n=2)$. For one deceased person, the cause of death was documented as a result of the ED without further specification, and for three others, the cause of death could not be obtained from the database.

With regard to the deaths in relation to age groups, none of patients between 10 and 19 years $(n=249)$ of age died. Half of the deaths $(n=9)$ happened in patients aged 30 and over, even though this age group accounted for only about $35 \%$ of people in this study, whereas $65 \%$ of the study participants were younger than 30 years. Table 1 provides further details about the frequency of deaths and psychiatric comorbidities in each age group.

\section{Standardised mortality ratio (SMR)}

The SMR for our study cohort was 2.52 (95\% CI 1.49, 3.97).

\section{Univariate analysis by Cox regressions}

Cox regressions revealed significant associations of mortality with older age, male gender and the presence of recorded SUD, AD and PD (Table 2).

\section{Multivariable analyses}

Controlling for age, sex, ethnicity, the residing borough, marital status and deprivation score as confounders, multivariable analysis outcomes by Cox regression are shown in Table 3. In these models, recorded PD remained significantly associated with mortality in patients with BN (adjusted HR $=3.36,95 \% \mathrm{CI} 1.05,10.73$ ), while associations with SUD (adjusted HR $=3.12$, 95\% CI: 0.81, 12.08) and $\mathrm{AD}$ (adjusted $\mathrm{HR}=2.47,95 \% \mathrm{CI} 0.90,6.78$ ) were diluted in strength and no longer statistically significant. Detailed information regarding multivariable analysis outcomes for $\mathrm{AD}$, SUD and PD as the major exposures of interest are presented separately in Supplementary Tables 1-3.

\section{Discussion}

\section{Main findings}

Of the 1501 patients with BN, 18 died during the more than 9-year observation period. A more than 2.5-fold increased risk of general mortality was found after age and gender standardization, compared to the general population in England and Wales. Within the univariate analysis of the study cohort, age and male sex was found associated with an 
Table 2 Univariate analysis on demographics and clinical characteristics for mortality among people with $\mathrm{BN}$ by $\mathrm{Cox}$ regressions $(N=1501)$

\begin{tabular}{|c|c|c|c|c|}
\hline Variables & $\begin{array}{l}\text { Mean } \pm \text { SD/num- } \\
\text { bers ( } \% \text { in column })\end{array}$ & $\begin{array}{l}\text { Number of } \\
\text { deaths (\% in } \\
\text { row) }\end{array}$ & Crude hazard ratio $(95 \% \mathrm{CI})$ & $p$ value \\
\hline Age (years old) & $28.54 \pm 9.48$ & - & $1.06(1.02,1.10)$ & 0.005 \\
\hline \multicolumn{5}{|l|}{ Gender } \\
\hline Female & $1417(94.40 \%)$ & $14(0.99 \%)$ & Ref & \\
\hline Male & $84(5.60 \%)$ & $4(4.76 \%)$ & $5.41(1.78,16.46)$ & 0.003 \\
\hline \multicolumn{5}{|l|}{ Marital status } \\
\hline Single & $1070(71.29 \%)$ & $13(1.21 \%)$ & Ref & \\
\hline Married/partner & $197(13.12 \%)$ & $0(0.00 \%)$ & 0.00 & 1.000 \\
\hline Divorced/separated & $51(3.40 \%)$ & $1(1.96 \%)$ & $1.36(1.18,10.40)$ & 0.768 \\
\hline Others & $183(12.19 \%)$ & $4(2.19 \%)$ & $1.33(0.43,4.12)$ & 0.616 \\
\hline \multicolumn{5}{|l|}{ Borough } \\
\hline Lambeth & $362(24.12 \%)$ & $5(1.38 \%)$ & Ref & \\
\hline Croydon & $183(12.19 \%)$ & $2(1.09 \%)$ & $0.73(0.14,3.75)$ & 0.704 \\
\hline Lewisham & $213(14.19 \%)$ & $4(1.88 \%)$ & $1.28(0.35,4.79)$ & 0.709 \\
\hline Southwark & $240(15.99 \%)$ & $1(0.42 \%)$ & $0.29(0.03,2.46)$ & 0.256 \\
\hline Bromley & $165(10.99 \%)$ & $2(1.21 \%)$ & $0.91(0.18,4.74)$ & 0.920 \\
\hline Bexley & $85(5.66 \%)$ & $0(0.00 \%)$ & - & - \\
\hline Greenwich & $99(6.60 \%)$ & $0(0.00 \%)$ & - & - \\
\hline Others & $154(10.26 \%)$ & $4(2.60 \%)$ & $1.45(0.39,5.42)$ & 0.579 \\
\hline \multicolumn{5}{|l|}{ Ethnicity } \\
\hline White & $1165(77.61 \%)$ & $11(0.94 \%)$ & Ref & \\
\hline Black & $88(5.86 \%)$ & $3(3.41 \%)$ & $3.41(0.95,12.21)$ & 0.060 \\
\hline Others/mixed & $248(16.52 \%)$ & $4(1.61 \%)$ & $1.76(0.56,5.52)$ & 0.335 \\
\hline \multicolumn{5}{|l|}{ Deprivation score } \\
\hline $1.56-21.03$ & $494(32.91 \%)$ & $5(1.01 \%)$ & Ref & \\
\hline $21.04-30.70$ & $494(32.91 \%)$ & $6(1.21 \%)$ & $1.20(0.37,3.92)$ & 0.766 \\
\hline $30.71-100$ & $496(33.04 \%)$ & $6(1.21 \%)$ & $1.24(0.38,4.07)$ & 0.720 \\
\hline Missing & $17(1.13 \%)$ & $1(5.88 \%)$ & $6.22(0.73,53.32)$ & 0.095 \\
\hline \multicolumn{5}{|c|}{ Substance use disorder } \\
\hline No & $1440(95.94 \%)$ & $15(1.04 \%)$ & Ref & \\
\hline Yes & $61(4.06 \%)$ & $3(4.92 \%)$ & $4.01(1.16,13.85)$ & 0.028 \\
\hline \multicolumn{5}{|l|}{ Affective disorders } \\
\hline No & $1259(83.88 \%)$ & $11(0.87 \%)$ & Ref & \\
\hline Yes & $242(16.12 \%)$ & $7(2.89 \%)$ & $3.30(1.28,8.51)$ & 0.014 \\
\hline \multicolumn{5}{|l|}{ Personality disorder } \\
\hline No & $1370(91.27 \%)$ & $13(0.95 \%)$ & Ref & \\
\hline Yes & $131(8.73 \%)$ & $5(3.82 \%)$ & $3.88(1.38,10.89)$ & 0.010 \\
\hline
\end{tabular}

Table 3 Multivariable analysis on psychiatric comorbidity for mortality among people with $\mathrm{BN}$ by Cox regressions $(N=1501)$, controlling age, sex, ethnicity, borough, marital status and deprivation score as confounders

\begin{tabular}{lll}
\hline Comorbidity & Adjusted hazard ratio $(95 \% \mathrm{CI})$ & $p$ value \\
\hline Affective disorder & $2.47(0.90,6.78)$ & 0.08 \\
Substance use disorder & $3.12(0.81,12.08)$ & 0.10 \\
Personality disorder & $3.36(1.05,10.73)$ & 0.04 \\
\hline
\end{tabular}

increased mortality. With confounders properly considered, the deceased patients suffered from PD significantly more often.

\section{Comparison to previous research}

SMRs of around 2 have been reported by previous research [24]. This figure might be even higher in certain populations of people with BN, for example, people within the first year after discharge from inpatient treatment [21].

Also, in accordance with our results, age is usually positively associated with mortality in patients with EDs in the 
literature [20,49]. We found an association of male sex with increased mortality in patients with BN. In general, an association of male sex with increased mortality is well characterised in the literature, and potential explanations relate to the different effects of sex hormones [50,51], the undertaking of hazardous behaviour [51] and the disadvantage of having only one $X$ chromosome [50-53]. The higher mortality of men among patients with EDs [54-56] may be related to the fact that EDs are often considered as a female disorder and thus stigmatised and underdiagnosed in men, and it may also be related to findings of greater general psychopathology in men with EDs [57-61].

In our study sample, the proportions of people with comorbid psychiatric disorders are rather low as compared to similar studies. Whereas we found a documented percentage of PD of about $9 \%$ in this group of BN patients (see Table 1), a recent meta-analysis obtained a proportion of $54 \%$ of comorbid PDs in patients with BN [12]. Nevertheless, one has to keep in mind that our data did not derive from standardised interviews covering the whole spectrum of psychopathology. In fact, in our study, PD diagnosis means a diagnosis established and documented by the responsible clinician. One can assume that this was only documented, if it was clinically relevant.

\section{Psychiatric comorbidity and mortality}

In our dataset, the presence of a PD contributed significantly to mortality in patients with $\mathrm{BN}$ even when controlling for covariates. This is in accordance with previous literature, where PD has been reported to contribute to mortality [32]. Associations of mortality with SUD and AD were not significant in the fully adjusted model. Therefore, our discussion will focus on PD.

Even though we would like to keep the discussion of mechanisms to a minimum, since we did not study mechanisms in your paper, we will try to interpret the obtained association between increased mortality and PDs in patients with BN. It could be explained by increased risk-taking behaviour and self-injuries on the basis of high impulsivity, which is often seen in BN [62, 63]. Specific personality traits such as impulsivity could further lead to the use of alcohol and drugs and, in turn, increase morbidity in this patient group [49, 64, 65]. Additionally, the consequences of both BN symptoms, such as vomiting, in combination with substance abuse sequelae, such as mucosa damage in the gastrointestinal system due to alcohol intake, could lead to potentially deadly medical conditions, such as oesophageal adenocarcinomas [66].

\section{Clinical implications}

Our results advise to be aware of the increased mortality risk of older patients, patients with PDs, and men with BN. For further clarification of the presence of a PD in clinical practice, screening tools and clinical interviews are available such as the SCID-II Questionnaire (SCID-II-PQ) and SCIDII interview [67] for PDs.

\section{Strengths and limitations}

The main strengths of this study are the study design and the size of cohort using CRIS as our data source. CRIS is one of the largest collections of users for secondary mental healthcare services in Western Europe. Using these data, with a retrospective cohort study, we identified a large number of 1501 patients with BN. Eighteen patients with BN died in the observation period, since study subjects were fairly young. Therefore, statistical power is limited.

When considering systematic errors and generalisability of these findings, the research outcomes might be only generalizable to secondary and tertiary health care settings. Our study is a clinical cohort study. It did not include patients with BN who did not seek help, who were solely treated by their general practitioners, who were not correctly diagnosed with BN by their primary health care provider and subsequently referred to SLaM or who did not have access to SLaM due to funding issues.

This clinical cohort study included participants who were already suffering from BN. People in this cohort had not been included into the study before the appearance of BN like they would have been in a population-based cohort study. This means that we do not have a completely comparable control group of people without BN that would only differ in terms of the presence of BN. Strictly speaking, we compared people with BN from South London to the general population of England and Wales to calculate the SMR, because this was the most comparable data available.

\section{Future research directions}

In the current project, we calculated the SMR for people with BN, and we identified certain demographic variables and PDs as risk factors increasing mortality in patients with $\mathrm{BN}$. We also reviewed the cause of death for the 18 patients who died in the observation window. Due to the small number of people who died in our study, future studies with a larger sample size and a longer observation period need to statistically confirm, whether lethal intoxication from drugs or alcohol, suicide, circulatory/heart failure and respiratory failure are really the top four killers for patients with $\mathrm{BN}$.

Additionally, it would be clinically relevant to find out, whether death by these causes could be predicted based on 
certain psychopathological features such as the presence of PD symptoms, based on physical comorbidities or on laboratory parameters. Such clinical chemical parameters predicting lethal intoxication with alcohol could be biological markers of alcohol abuse such as gamma-glutamyl transferase (GGT), mean corpuscular volume (MCV), carbohydrate-deficient transferrin (CDT) or high-density lipoprotein (HDL) cholesterol [68, 69]. Alterations in the biological stress systems and the serotonergic system could be tested as predictors of suicidal behaviour [70]. And serum electrolyte concentrations which are often disturbed in patients with BN due to vomiting [71, 72] could be examined as biomarkers predicting circulatory, heart or respiratory failure.

Once the main causes of death and ways to predict them with a certain probability are established for patients with $\mathrm{BN}$, this knowledge should be clinically applied for the individualised prevention of premature death. Such research projects could involve frequent monitoring of the mental and physical health state as well as the measurement of certain biomarkers. As PDs contributed significantly to the mortality risk of people with $\mathrm{BN}$ in the present study, clinical projects to develop and test therapies for patients with both BN and PDs would also seem sensible to find ways to prevent premature death in this patient group. Small clinical pilot studies in patients with $\mathrm{BN}$ and comorbid PD using dialectical behaviour therapy (DBT) have already been performed and published [73, 74]. However, the low remission rates and the maintained eating-related psychopathology suggest that those treatments need further improvement [74].

Taken together, future research should establish the main causes of death for people with BN, seek to predict the causes of death by focussing on physical comorbidities and biomarkers and try to develop specific therapies for people with both BN and PDs. This means that epidemiological, biomarker and therapy research need to cooperate to achieve further scientific and clinical progress.

Funding This work was supported by the Clinical Records Interactive Search (CRIS) system funded and developed by the National Institute for Health Research (NIHR) Biomedical Research Centre (BRC) at South London and Maudsley NHS Foundation Trust (SLaM) and King's College London and a joint infrastructure grant from Guy's and St Thomas' Charity and the Maudsley Charity (grant number BRC2011-10035). RH, RS, HS, C-KC, US and JT have received salary support from the NIHR BRC at SLaM and King's College London. The views expressed are those of the authors and not necessarily those of the NHS, the NIHR or the Department of Health.

\section{Compliance with ethical standards}

Conflict of interest RH, C-KC, HS, and RS have received research funding from Roche, Pfizer, Janssen, and Lundbeck. MH has received research funding from Janssen, Merck, Lundbeck, UCB and Biogen as part of the RADAR-CNS consortium-a public private partnership funded under the European Commission's Innovative Medicines Initiative.
OpenAccess This article is distributed under the terms of the Creative Commons Attribution 4.0 International License (http://creativeco mmons.org/licenses/by/4.0/), which permits unrestricted use, distribution, and reproduction in any medium, provided you give appropriate credit to the original author(s) and the source, provide a link to the Creative Commons license, and indicate if changes were made.

\section{References}

1. American Psychiatric Association (1980) Diagnostic diagnostic and statistical manual of mental disorders and statistical manual of mental disorders, 3rd edn. APA Publishing, Washington, DC

2. Russell G (1979) Bulimia nervosa: an ominous variant of anorexia nervosa. Psychol Med 9:429-448

3. American Psychiatric Association (2013) Diagnostic and statistical manual of mental disorders, 5th edn. APA Publishing, Arlington

4. World Health Organization (1992) Manual of the international statistical classification of diseases and related health problems 10th revision (ICD-10). WHO, Geneva

5. Zerwas S, Larsen JT, Petersen L et al (2015) The incidence of eating disorders in a Danish register study: associations with suicide risk and mortality. J Psychiatr Res 65:16-22

6. Keski-Rahkonen A, Mustelin L (2016) Epidemiology of eating disorders in Europe: prevalence, incidence, comorbidity, course, consequences, and risk factors. Curr Opin Psychiatry 29:340-345

7. Miniati M, Benvenuti A, Bologna E et al (2018) Mood spectrum comorbidity in patients with anorexia and bulimia nervosa. Eat Weight Disord 23:305-311

8. Ulfvebrand $\mathrm{S}$, Birgegård $\mathrm{A}$, Norring $\mathrm{C}$ et al (2015) Psychiatric comorbidity in women and men with eating disorders results from a large clinical database. Psychiatry Res 230:294-299

9. Dansky BS, Brewerton TD, Kilpatrick DG (2000) Comorbidity of bulimia nervosa and alcohol use disorders: results from the National Women's Study. Int J Eat Disord 27:180-190

10. Fouladi F, Mitchell JE, Crosby RD et al (2015) Prevalence of alcohol and other substance use in patients with eating disorders. Eur Eat Disord Rev 23:531-536

11. Wade T, Bulik CM, Prescott CA, Kendler KS (2004) Sex influences on shared risk factors for bulimia nervosa and other psychiatric disorders. Arch Gen Psychiatry 61:251-256

12. Martinussen M, Friborg O, Schmierer P et al (2017) The comorbidity of personality disorders in eating disorders: a meta-analysis. Eat Weight Disord 22:201-209

13. Sansone RA, Levitt JL, Sansone LA (2006) The prevalence of personality disorders in those with eating disorders. In: Sansone RA, Levitt JL (eds) Personality disorders and eating disorders: exploring the frontier. Routledge, New York, pp 23-39

14. Herzog DB, Nussbaum KM, Marmor AK (1996) Comorbidity and outcome in eating disorders. Psychiatr Clin North Am 19:843-859

15. Del Pino-Gutiérrez A, Jiménez-Murcia $S$, Fernández-Aranda $F$ et al (2017) The relevance of personality traits in impulsivityrelated disorders: from substance use disorders and gambling disorder to bulimia nervosa. J Behav Addict 6:396-405

16. Lilenfeld LR, Kaye WH, Greeno CG et al (1997) Psychiatric disorders in women with bulimia nervosa and their first-degree relatives: effects of comorbid substance dependence. Int J Eat Disord 22:253-264

17. Vitousek K, Manke F (1994) Personality variables and disorders in anorexia nervosa and bulimia nervosa. J Abnorm Psychol 103:137-147 
18. Hadad NA, Knackstedt LA (2014) Addicted to palatable foods: comparing the neurobiology of bulimia nervosa to that of drug addiction. Psychopharmacology 231:1897-1912

19. Pearson CM, Lavender JM, Cao L et al (2017) Associations of borderline personality disorder traits with stressful events and emotional reactivity in women with bulimia nervosa. $\mathrm{J}$ Abnorm Psychol 126:531-539

20. Fichter MM, Quadflieg N (2016) Mortality in eating disordersresults of a large prospective clinical longitudinal study. Int $\mathbf{J}$ Eat Disord 49:391-401

21. Hoang U, Goldacre M, James A (2014) Mortality following hospital discharge with a diagnosis of eating disorder: national record linkage study, England, 2001-2009. Int J Eat Disord 47:507-515

22. National Institute for Health and Care Excellence (NICE) (2017) Eating disorders: recognition and treatment. https://www.nice.org. uk/guidance/ng69/resources/eating-disorders-recognition-andtreatment-pdf-1837582159813. Accessed 24 Jan 2019

23. Deutsche G (2010) Psychosomatische Medizin und Psychotherapie und das Deutsche Kollegium für Psychosomatische Medizin: S3-Leitlinie "Essstörungen, Diagnostik und Therapie", 2010. http://www.awmf.org/leitlinien/detail/11/051-026.html. Accessed 24 Jan 2019

24. Arcelus J, Mitchell AJ, Wales J, Nielsen S (2011) Mortality rates in patients with anorexia nervosa and other eating disorders. A meta-analysis of 36 studies. Arch Gen Psychiatry 68:724-731

25. Brown CA, Mehler PS (2013) Medical complications of selfinduced vomiting. Eat Disord 21:287-294

26. Forcano L, Fernández-Aranda F, Alvarez-Moya E et al (2009) Suicide attempts in bulimia nervosa: personality and psychopathological correlates. Eur Psychiatry 24:91-97

27. Pisetsky EM, Thornton LM, Lichtenstein P et al (2013) Suicide attempts in women with eating disorders. J Abnorm Psychol 122:1042-1056

28. Tachi T, Murakami K, Murotsu K, Washizuka T (2001) Affective states associated with bingeing and purging behaviours in Japanese patients with bulimia nervosa. Br J Med Psychol 74(Pt 4):487-496

29. Whitfield JB, Heath AC, Madden PAF (2018) Effects of high alcohol intake, alcohol-related symptoms and smoking on mortality. Addiction 113:158-166

30. Willmore J, Marko TL, Taing D, Sampasa-Kanyinga H (2017) The burden of alcohol-related morbidity and mortality in Ottawa, Canada. PLoS One 12:e185457

31. Degenhardt L, Bucello C, Mathers B et al (2011) Mortality among regular or dependent users of heroin and other opioids: a systematic review and meta-analysis of cohort studies. Addiction 106:32-51

32. Cailhol L, Pelletier É, Rochette L et al (2017) Prevalence, mortality, and health care use among patients with cluster $b$ personality disorders clinically diagnosed in Quebec: a provincial cohort study, 2001-2012. Can J Psychiatry 62:336-342

33. Ho CSH, Jin A, Nyunt MS et al (2016) Mortality rates in major and subthreshold depression: 10-year follow-up of a Singaporean population cohort of older adults. Postgrad Med 128:642-647

34. Mitchell JE, Specker SM, de Zwaan M (1991) Comorbidity and medical complications of bulimia nervosa. J Clin Psychiatry 52 Suppl:13-20

35. O'Brien KM, Vincent NK (2003) Psychiatric comorbidity in anorexia and bulimia nervosa: nature, prevalence, and causal relationships. Clin Psychol Rev 23:57-74

36. Duncan AE, Neuman RJ, Kramer J et al (2005) Are there subgroups of bulimia nervosa based on comorbid psychiatric disorders? Int J Eat Disord 37:19-25

37. Keel PK, Dorer DJ, Eddy KT et al (2003) Predictors of mortality in eating disorders. Arch Gen Psychiatry 60:179-183
38. Keel PK, Mitchell JE, Miller KB et al (1999) Long-term outcome of bulimia nervosa. Arch Gen Psychiatry 56:63-69

39. Kjær JNR, Biskin R, Vestergaard C et al (2018) All-cause mortality of hospital-treated borderline personality disorder: a nationwide cohort study. J Pers Disord 11:1-13

40. Patten SB, Williams JV, Bulloch AG (2019) Major depressive episodes and mortality in the Canadian household population. J Affect Disord 242:165-171

41. Miloyan B, Bulley A, Bandeen-Roche K et al (2016) Anxiety disorders and all-cause mortality: systematic review and metaanalysis. Soc Psychiatry Psychiatr Epidemiol 51:1467-1475

42. Suokas JT, Suvisaari JM, Gissler M (2013) Mortality in eating disorders: a follow-up study of adult eating disorder patients treated in tertiary care, 1995-2010. Psychiatry Res 210:1101-1106

43. Pan YJ, Yeh LL, Chen YC et al (2016) Hospital treatment, mortality and heathcare costs in relation to socioeconomic status among people with bipolar affective disorder. BJPsych Open 2:10-17

44. Perera G, Broadbent M, Callard F et al (2016) Cohort profile of the South London and Maudsley NHS Foundation Trust Biomedical Research Centre (SLaM BRC) Case Register: current status and recent enhancement of an electronic mental health recordderived data resource. BMJ Open 6:e008721

45. Stewart R, Soremekun M, Perera G et al (2009) The South London and Maudsley NHS Foundation Trust Biomedical Research Centre (SLAM BRC) case register: development and descriptive data. BMC Psychiatry 9:51

46. Chang CK, Hayes RD, Broadbent MT et al (2014) A cohort study on mental disorders, stage of cancer at diagnosis and subsequent survival. BMJ Open 4:e004295

47. Su YP, Chang CK, Hayes RD et al (2014) Mini-mental state examination as a predictor of mortality among older people referred to secondary mental healthcare. PLoS One 9:e105312

48. Hayes RD, Downs J, Chang CK et al (2015) The effect of clozapine on premature mortality: an assessment of clinical monitoring and other potential confounders. Schizophr Bull 41:644-655

49. Ackard DM, Richter S, Egan A, Cronemeyer C (2014) Poor outcome and death among youth, young adults, and midlife adults with eating disorders: an investigation of risk factors by age at assessment. Int J Eat Disord 47:825-835

50. Waldron I (1993) Recent trends in mortality ratios for adults in developed countries. Soc Sci Med 36:451-462

51. Gjonça A, Tomassini C, Toson B, Smallwood S (2005) Sex differences in mortality, a comparison of the United Kingdom and other developed countries. Health Stat Q 26:6-16

52. Christensen K, Kristiansen M, Hagen-Larsen H et al (2000) $\mathrm{X}$-linked genetic factors regulate hematopoietic stem-cell kinetics in females. Blood 95:2449-2450

53. Waldron I (1995) Contributions of biological and behavioural factors to changing sex differences in ischaemic heart disease mortality. In: Lopez AD, Caselli G, Volkonen T (eds) Adult mortality in developed countries: from description to explanation. Clarendon Press, Oxford, pp 161-178

54. Fernández-Aranda F, Aitken A, Badía A et al (2004) Personality and psychopathological traits of males with an eating disorder. Eur Eat Disord Rev 12:367-374

55. Gueguen J, Godart N, Chambry J et al (2012) Severe anorexia nervosa in men: comparison with severe AN in women and analysis of mortality. Int J Eat Disord 45:537-545

56. Mitchison D, Mond J (2015) Epidemiology of eating disorders, eating disordered behaviour, and body image disturbance in males: A narrative review. J Eat Disord 3:20

57. Núñez-Navarro A, Agüera Z, Krug I et al (2012) Do men with eating disorders differ from women in clinics, psychopathology and personality? Eur Eat Disord Rev 20:23-31 
58. Griffiths S, Mond JM, Li Z et al (2015) Self-stigma of seeking treatment and being male predict an increased likelihood of having an undiagnosed eating disorder. Int J Eat Disord 48:775-778

59. Strother E, Lemberg R, Stanford SC, Turberville D (2012) Eating disorders in men: underdiagnosed, undertreated, and misunderstood. Eat Disord 20:346-355

60. Weltzin TE, Weisensel N, Franczyk D et al (2005) Eating disorders in men: update. J Men Health Gend 2:186-193

61. Agüera Z, Sánchez I, Granero R et al (2017) Short-term treatment outcomes and dropout risk in men and women with eating disorders. Eur Eat Disord Rev 25:293-301

62. Fichter MM, Quadflieg N, Hedlund S (2008) Long-term course of binge eating disorder and bulimia nervosa: relevance for nosology and diagnostic criteria. Int J Eat Disord 41:577-586

63. Keel PK, Mitchell JE (1997) Outcome in bulimia nervosa. Am J Psychiatry 154:313-321

64. Conason AH, Brunstein Klomek A, Sher L (2006) Recognizing alcohol and drug abuse in patients with eating disorders. QJM 99:335-339

65. Franko DL, Keshaviah A, Eddy KT et al (2013) A longitudinal investigation of mortality in anorexia nervosa and bulimia nervosa. Am J Psychiatry 170:917-925

66. Brewster DH, Nowell SL, Clark DN (2015) Risk of oesophageal cancer among patients previously hospitalised with eating disorder. Cancer Epidemiol 39:313-320

67. Ekselius L, Lindström E, von Knorring L et al (1994) SCID II interviews and the SCID Screen questionnaire as diagnostic tools for personality disorders in DSM-III-R. Acta Psychiatr Scand 90:120-123

68. Andresen-Streichert H, Müller A, Glahn A et al (2018) Alcohol biomarkers in clinical and forensic contexts. Dtsch Arztebl Int 115:309-315

69. Szegedi A, Müller MJ, Himmerich H et al (2000) Carbohydratedeficient transferrin (CDT) and HDL cholesterol (HDL) are highly correlated in male alcohol dependent patients. Alcohol Clin Exp Res 24:497-500

70. Oquendo MA, Sullivan GM, Sudol K et al (2014) Toward a biosignature for suicide. Am J Psychiatry 171:1259-1277

71. Crow SJ, Salisbury JJ, Crosby RD et al (1997) Serum electrolytes as markers of vomiting in bulimia nervosa. Int J Eat Disord 21:95-98

72. Franke S, Halfter SM, Himmerich H (2010) Diagnosis and therapy of bulimia nervosa. MMW Fortschr Med 152:80-82

73. Chen EY, Matthews L, Allen C et al (2008) Dialectical behavior therapy for clients with binge-eating disorder or bulimia nervosa and borderline personality disorder. Int J Eat Disord 41:505-512

74. Kröger C, Schweiger U, Sipos V et al (2010) Dialectical behaviour therapy and an added cognitive behavioural treatment module for eating disorders in women with borderline personality disorder and anorexia nervosa or bulimia nervosa who failed to respond to previous treatments. An open trial with a 15-month follow-up. J Behav Ther Exp Psychiatry 41:381-388 